\title{
Reproducibility of the Reading Imperial Surrey Saturated fat Cholesterol Intervention (RISSCI-1 and 2) study
}

\author{
A. Koutsos ${ }^{1}$, R. Antoni ${ }^{2}$, G. Wong ${ }^{1}$, L. Sellem ${ }^{1}$, E. Ozen ${ }^{1}$, H. Ayyad ${ }^{2}$, B.A. Fielding ${ }^{2}$, \\ M.D. Robertson ${ }^{2}$, K.G. Jackson ${ }^{1}$, J. Mendis ${ }^{2}$, N. Jackson ${ }^{2}$, B.A. Griffin ${ }^{2}$ and J.A. Lovegrove ${ }^{1}$ \\ ${ }^{1}$ Hugh Sinclair Unit of Human Nutrition, Department of Food and Nutritional Sciences, University of Reading, \\ Whiteknights, Reading, UK and \\ ${ }^{2}$ Department of Nutritional Sciences, University of Surrey, Guildford, UK
}

The reproducibility of clinical trial results is of major importance in nutrition research. Variability in the results of apparently similar dietary studies has been previously reported ${ }^{(1)}$, due to differences in study design, assessment methods, participants' background diet and inter-individual variability. However, comparisons of identical dietary interventions performed in the same participants, which will control for some of the mentioned variability, are rare. This study aims to assess the reproducibility of an identical dietary intervention (Reading, Imperial, Surrey Saturated fat Cholesterol Intervention study 1 and 2, RISSCI-1 and RISSCI-2) performed on two occasions, on the outcomes of fasted lipids.

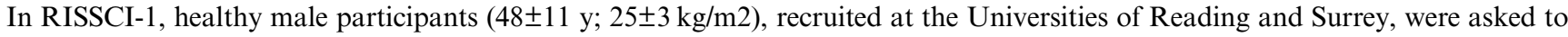
follow a high saturated fatty acid diet (SFA) (18\% total energy) followed by a lower SFA diet (10\% total energy), each for 4 weeks. The diets were isoenergetic and dietary SFA was replaced with unsaturated fatty acids (UFAs) using a food exchange model ${ }^{(2,3)}$. The 8-week sequential, non-randomised study, included three visits; visit 1 as a baseline, and visits 2 and 3 after the high and the lower SFA diet, respectively. Thirty-six volunteers were selected to run the same dietary intervention (RISSCI-2). A General Linear Mixed Model was performed using the Mixed Procedure in SAS ${ }^{\circledR}$ (Proc Mixed) to explore the reproducibility of the intervention. The change in serum lipids (total cholesterol (TC), low density lipoprotein-cholesterol (LDL-C), high density lipoprotein-cholesterol (HDL-C) and triacylglycerol) between the high and the lower SFA diet was used as the outcome measure and the change between the baseline and the high SFA diet as a covariate. The study, categorical variable (RISSCI-1, RISSCI-2), was used as a fixed effect. Subjects nested within the study centre (Reading, Surrey) were used as random effects. Comparisons between the high and the lower SFA diet were also performed using a paired t-test.

There is no evidence to demonstrate that the responsiveness of the fasting lipids to the replacement of dietary SFAs with UFAs performed on two occasions (RISSCI-1 and 2), was significantly different (TC $(\mathrm{P}=0.992)$, LDL-C $(\mathrm{P}=0.545)$, HDL-C $(\mathrm{P}=0.724)$ and triacylglycerol $(\mathrm{P}=0.272)$ ). Replacement of dietary SFAs with UFAs reduced TC by $12.6 \%$ in RISSCI- 1 and by $12 \%$ in RISSCI-2 (mean differences $\pm \mathrm{SD}, 0.66 \pm 0.50$ and $0.61 \pm 0.57 \mathrm{mmol} / \mathrm{L}$ respectively, $\mathrm{P}<0.001$ ). Similarly, LDL-C reduced by $16.8 \%$ and $15.6 \%$ respectively (mean differences $\pm \mathrm{SD}, 0.55 \pm 0.42$ and $0.49 \pm 0.45 \mathrm{mmol} / \mathrm{L}$ respectively, $\mathrm{P}<0.001$ ). HDL-C reduced by $6.4 \%$ and $4.7 \%$, respectively (mean differences $\pm \mathrm{SD}, 0.095 \pm 0.13$ and $0.065 \pm 0.14 \mathrm{mmol} / \mathrm{L}$, respectively, $\mathrm{P}<0.001$ ).

This preliminary analysis indicates that responsiveness of the fasting lipid profile to the replacement of SFAs with UFAs was similar for RISSCI-1 and 2. Reproducibility in response to dietary interventions is important in determining the efficacy of public dietary recommendations.

\section{Acknowledgements}

The RISSCI study was funded by the Biotechnology \& Biological Sciences Research Council (Grant no: BB/P010245/1).

\section{References}

1. Sorkin BC, Kuszak AJ, Williamson JS, et al. (2016) Adv Nutr 7, 383-389.

2. Antoni R, Sellem L, Koutsos A, et al. (2019) Proc Nutr Soc 78, (OCE1), E9.

3. Koutsos A, Antoni R, Ozen E, et al. (2020) Proc Nutr Soc 79, (OCE1), E6. 